\title{
Capturing nitrosamines in aqueous solution by composited super-hydrophobic silicic xerogel
}

Shuo Hao Li ${ }^{1,2}$, Wei Gang Lin ${ }^{1,2}$,Bi Cheng Huang ${ }^{1}$, Lei-jun Wang ${ }^{2}$, Wen-bo $G u^{2}$, Wei-miao Wang ${ }^{2}$, Zheng Yu Yang ${ }^{2 *}$, Ying Wang ${ }^{3}$, Jian Hua Zhu ${ }^{1^{*}}$

${ }^{1}$ Key Laboratory of Mesoscopic Chemistry of MOE, College of Chemistry and Chemical Engineering, Nanjing University, Nanjing 210023, China, E-mail: jhzhu@ nju.edu.cn

${ }^{2}$ Technology Center of Shanghai Tobacco (Group) Company, Shanghai 200082, China, E-mail: zyyang@sh.tobacco.com.cn

${ }^{3}$ College of Chemistry and Chemical Engineering, Nanjing University, Nanjing 210023, China

\begin{abstract}
Super-hydrophobic silicic xerogel (SG) was prepared from methyltrimethoxysilane (MTMS) and dimethyldimethoxysilane (DMDMS), and the optimized MTMS to DMDMS molar ratio was determined to be $3 / 2$. To fabricate new effective nitrosamines sorbent in tobacco extract solution, SG was modified by epitaxial growth of mesoporous silica to form the coated composite named as MSGx with a large surface area $\left(152-208 \mathrm{~m}^{2} \mathrm{~g}^{-1}\right)$ and pore volume $\left(0.133-0.162 \mathrm{mLg}^{-1}\right)$, and the new sorbent could capture 54 59\% of nitrosamines in tobacco solution, superior to zeolite and mesoporous silica.
\end{abstract}

Keywords Super-hydrophobic silicic xerogel; epitaxial growth of mesoporous silica; nitrosamines; liquid adsorption; environment protection 


\section{Introduction}

Nitrosamines are deemed to be the widespread carcinogens characterized with $N$-nitroso group, including volatile nitrosamines (VNA), non-volatile nitrosamines and tobacco specific nitrosamines (TSNA). TSNA are a group of carcinogens found only in tobacco products. They can cause various cancers or tumors in laboratorial animals and possess the potential carcinogenicity to human though their content in tobacco and tobacco products is in the range of $\mu \mathrm{g} \mathrm{g}^{-1}$ (ppm) or $\mathrm{ng} \mathrm{g}^{-1}(\mathrm{ppb})$. Among various efforts controlling the pollution of nitrosamines in environment, one strategy is to adsorb TSNA in tobacco extract solution in order to reduce the injurant at source [1-4], and effective sorbent is the key. TSNA are oily molecules and their adsorption in tobacco extract solution is inevitably interfered by water [1], so the favorable separation performance between water and oil is vital. Nowadays, many porous hydrophobic materials have been extensively studied [5], including carbon based materials [6], metal oxide nanowires [7, 8], biological nanometer fiber $[9,10]$, organic polymer $[11,12]$ and macroporous aerogel [13-15], etc. However, complex synthesis and high cost hinders their actual application.

Recently, hydrophobic polymethylsilsesquioxane (PMSQ) materials have been synthesized by crosslinking polymerization of methyltrimethoxysilane (MTMS), in which mesopores and macropore could be produced through phase separation $[16,17]$. Also, flexible marshmallow-like silicic aerogel/xerogel was synthesized from MTMS and dimethyldimethoxysilane (DMDMS) co-precursor [18]. This aerogel is expected to 
be the good trapper of organic substance in aqueous solution due to its inherent hydrophobicity. Hence, superhydrophobic silicic xerogel is prepared here by a optimizing the proportion of two silicon sources (MTMS and DMDMS), and used for capturing nitrosamines in tobacco extract solution.

Tobacco is a complex mixture of over 2000 chemical constituents [19], and tobacco-extract solution contains the nitrosamines with a low concentration of $\mu \mathrm{g} \mathrm{g}^{-1}$ (ppm) [4] whereas the abundant water solvent hampers the adsorption of organic carcinogens. To conquer this challenge, mesoporous silica MCM-48 was modified by 3-chloropropyltriethoxysilane to suppress the adsorption of water [1], while zinc oxide was inserted in activated carbon to strengthen the electrostatic attraction towards the N-NO group of nitrosamines [2,3]. Here the super hydrophobic silicic xerogel will be coated with mesoporous silica through epitaxial growth method to fabricate core-shell type composite, in order to enhance its capability of capturing nitrosamines in tobacco extract solution by means of synergy between the hydrophobic core and the hydrophilic mesoporous silica shell.

\section{Experimental section}

\subsection{Materials}

Cetyltrimethylammonium bromide (CTAB) was the product of Nanjing Chemical Reagents. Zeolite NaY and NaZSM-5 were commercially available powders, HZSM-5 was obtained by ion exchange from parent NaZSM-5 [20]. Mesoporous silica MCM-41, SBA-15 and MCM-48 were prepared according to literature [21-23]. Activated carbon 
(AC) was bought from Chemviron Carbon, and Table 1 lists the texture properties of these porous sorbents. Tobacco shred (HHM, Tianjin), the fine cut blended type tobacco with $4 \mathrm{~mm}$ length and 1-2 mm width, was purchased from market. Other reagents were with AR grade and used as received.

\subsection{Synthesis of super-hydrophobic silicic xerogel}

$1.82 \mathrm{~g} \mathrm{CTAB}$ and $10 \mathrm{~g}$ urea were firstly dissolved into $30 \mathrm{~g}$ acetic acid $(5 \mathrm{mM})$ and stirred at $303 \mathrm{~K}$ for $0.5 \mathrm{~h}$. Thereafter, $5.72 \mathrm{~g}$ MTMS and $3.36 \mathrm{~g}$ DMDMS were added in the solution and stirred for $0.5 \mathrm{~h}$ and then heated at $353 \mathrm{~K}$ for $24 \mathrm{~h}$. The obtained monolithic xerogel was filtered off, thoroughly washed with distilled water and methanol to remove $\mathrm{CTAB}$ and other unreacted reagents. Then, the solid was air-dried and ethanol extracted, giving the sample denoted as $\mathrm{SG}(\mathrm{r})$ where $\mathrm{r}$ was the molar ratio of MTMS and DMDMS.

\subsection{Synthesis of the silicic xerogel covered with epitaxially grown mesoporous silica}

$0.73 \mathrm{~g} \mathrm{CTAB}, 2.1 \mathrm{~g}$ citric acid and the sodium citrate with a calculated amount were dissolved in $60 \mathrm{~g}$ distilled water, and then $2.08 \mathrm{~g}$ tetraethoxysilane (TEOS) was put into the solution. After that, $0.5 \mathrm{~g} \mathrm{SG}(3 / 2)$ was added and slowly stirred for $48 \mathrm{~h}$. The resulting sample was filtrated, washed, dried and extracted as aforementioned, named as MSGx where $\mathrm{x}$ was the molar ratio of sodium citrate and citric acid. Besides, the sample named Mx was synthesized under the similar conditions but no silicic xerogel additive.

\subsection{Characterization}

Nitrogen adsorption-desorption isotherm was performed on a Micromeritics ASAP 
2020 volumetric adsorption analyzer at $77 \mathrm{~K}$. About $100 \mathrm{mg}$ of sample was evacuated at $573 \mathrm{~K}$ for $4 \mathrm{~h}$ in the degas port prior to test. Specific surface areas were calculated by the BET method using adsorption data acquired in the relative pressure range $\left(\mathrm{P} / \mathrm{P}_{0}\right)$ from 0.04 to 0.2 , while the total pore volume was determined from the amount adsorbed at a $\mathrm{P} / \mathrm{P}_{0}$ of about 0.99 . Adsorption isotherm of water was measured at $298 \mathrm{~K}$, and all samples were degassed at $373 \mathrm{~K}$ and $0.6 \mathrm{~Pa}$ for $2 \mathrm{~h}$ prior to adsorption. FTIR spectrum was recorded in the range of $400-4000 \mathrm{~cm}^{-1}$ on a NEXUS870 spectrometer, for which the sample was mixed with $\mathrm{KBr}$ at a ratio of 3:97 (w/w) and then pressed as a thin disk for testing. The morphology of sample was observed by SEM using a Hitachi S4800 FE-SEM system with $10 \mathrm{kV}$ accelerating voltage and $10 \mathrm{~mA}$ of beam current, and TEM analysis was carried out on a JEM-2100 electron microscope operating at $200 \mathrm{kV}$. Water contact angles were measured using the sessile drop method with a $3 \mu \mathrm{L}$ water droplet and a telescopic goniometer (Rame-Hart, Inc., MountainLake, NJ).

\subsection{Liquid adsorption in tobacco extract solution}

$100 \mathrm{~g}$ fine cut tobacco was submerged into $1 \mathrm{~L}$ distilled water that contained $6 \mathrm{~g}$ ascorbic acid, and the mixture was kept at $303 \mathrm{~K}$ for $24 \mathrm{~h}$ then filtrated to separate residue $[1,2]$. The obtained tobacco extract solution was stored at $277 \mathrm{~K}$. Liquid adsorption of nitrosamines was performed under dynamic condition [2]. $40 \mathrm{mg}$ granular adsorbent was added into $40 \mathrm{~mL}$ tobacco extract solution in cone flasks and stirred without light at $303 \mathrm{~K}$ for $1 \mathrm{~h}$. Thereafter it was centrifuged at $3000 \mathrm{rpm}$ for $15 \mathrm{~min}$ to separate solid and solution. 


\subsection{Determination of nitrosamines and TSNA in solution}

$30 \mathrm{~mL}$ tobacco extract solution was extracted with $30 \mathrm{~mL}$ dichloromethane for 4 times, and the organic components were concentrated to $25 \mathrm{~mL}$ after dried with anhydrous sodium sulfate. The total quantity of nitrosamines in the solution was determined by improved spectrophotometric method $[1,2,21]$, giving the data in $\mu$ mol $\mathrm{g}^{-1}$. The TSNA content of tobacco extract solution was determined by Applied Biosystems 4000 Q TRAP mass spectrum combined with an Agilent 1200 liquid chromatogram system equipped with a column of CAPCELL PAK C18 MG2 (4.6 $\mathrm{mm} \times 150 \mathrm{~mm}, 3 \mu \mathrm{m})$. And the procedure and parameters used in the detection were same as that reported previously $[2,24]$.

\section{Results}

\subsection{Synthesis of super-hydrophobic silicic xerogel}

MTMS/DMDMS molar ratio significantly affected the morphology of super-hydrophobic silicic xerogel. Absence of DMDMS in synthesis resulted in a sand-like sample. Small monoliths appeared once the molar ratio of MTMS/DMDMS achieved 4/1, and large monolith formed and perfected at the ratio of $3 / 2$ and $1 / 1$, nrespectively (Fig. S1). SG(3/2) sample had the optimal elastic and mechanical property so that subsequent studies were focused on SG (3/2) that was abbreviated as SG otherwise stated. Increasing the amount of CTAB and urea reduced the flexibility and appearances of products (Table S1), and their morphology was different (Fig. S2a, S2b, S2c). SG (3/2) samples had lots rounded colloid particles with smooth mellow surface 
and the size of microns [16], but the sample of $\mathrm{SG}(1 / 1)$ prepared with more urea was crisp and fragmented (Fig. S2d), on which lots of pores and flaws emerged (Fig. S2e and S2f). Replacement of CTAB with F127 changed the morphology of SG(r) sample from rounded colloid particles to sands-like sheets (Fig. S2h, S2i, Table S1), but substitution of METES with 3-methacryloxypropyl-triethoxysilane (MPTES) or 3-aminopropyltriethoxysilane (APTES) failed to get solid products (Table S1). After calcination at $823 \mathrm{~K}, \mathrm{SG}(\mathrm{r})$ samples formed crushed fragments with somewhere black and lost 22 67\% of weight (Table S2).

Super-hydrophobic silicic xerogel has an excellent elasticity since it has mesh skeleton of polydimethylsiloxane (PDMS) [7, 11]. Silicic xerogel shrinked under pressure, but it perfectly spring-back once pressure was removed (Fig. S3). This xerogel has super-hydrophobic surface, because of its rough surface resulting from the decomposition of meta-stable states [25], and the most groups exposed on its surface were methyl rather than hydroxyl. So, water droplet located on the surface of SG looked like a ball (Fig. S3) but methylene chloride completely infiltrated (Figure not shown). Water adsorption at $298 \mathrm{~K}$ confirmed the super hydrophobicity of SG sample (Fig.1a). Mesoporous silica SBA-15 adsorbed about $17 \%$ of water at the $\mathrm{p} / \mathrm{p}_{0}$ of 0.8 , but the SG trapped only $0.5 \%$ under the same condition (Fig. 1a).

\subsection{Synthesis of the xerogel covered with epitaxially grown mesoporous silica}

Silicic xerogel SG had a small surface area (Table 1) that surly limited its adsorption capabicity therefore it was coated with mesoporous silica by means of epitaxial growth 
technique (Scheme 1). SG sample floated on water but immersed in synthetic solution (Fig. S4), in which the surfactant CTAB was adhered on SG, followed by the interaction with and polycondensation of siliceous reactants (Scheme 1). SG had lots rounded colloid particles with smooth mellow surface and the size of several microns [16]. After the epitaxial growth process, it was coated by a layer of shell containing mesoporous silica (Fig. 2) with thickness of 50 100 nm. Nonetheless, the shell of MSG0.5 or MSG2.5 composites appeared cracks and thus formed multiple plates (Fig. S5) hence the surface of xerogel core inevitably exposed more or less. Actually, rigid mesoporous silica was difficult to completely fit in the xerogel due to the curvature of spherical surface, consequently many cracks generated (Fig. S6). Most of the mesoporous silica shell were smooth and homogeneous, but their thickness was different: on the sample of MSG0.5 it varied from $200 \mathrm{~nm}$ to $300 \mathrm{~nm}$ while the value was 100 200 $\mathrm{nm}$ on the MSG2.5 composite.

Figure 3 displayed TEM images of SG xerogel and MSGx composites. SG was opaque solid core (Fig. 3a and 3b) while a crustose layer of mesoporous silica coated on the colloidal particles in MSG0.5 composite (Fig. 3c). The shell thickness of MSG0.5 sample varied from 200 to $300 \mathrm{~nm}$ while it was about $100 \mathrm{~nm}$ on MSG2.5 composite, in good agreement with the results of SEM test. The surface of silicic xerogel was attached by the vermicular mesoporous silica without neat symmetry (Fig. 3d), and there was no obvious characteristic peak on the low angle XRD pattern, either (Fig. S7). However, their counterparts, both of M0.5 and M2.5 were the ordered mesoporous materials [26]. 
No doubt, the hydrophobic surface of xerogel influences arrangement of template micelles along with the symmetry of mesoporous structure.

Table 1 listed the structural properties of SG, MSGx and other related porous materials. SG had only $4 \mathrm{~m}^{2} \cdot \mathrm{g}^{-1}$ of specific surface because of its imporous structure, but MSG0.5 and MSG2.5 had a dramatically enlarged specific surface area and pore volume, $208 \mathrm{~m}^{2} \cdot \mathrm{g}^{-1}$ and $0.16 \mathrm{~cm}^{3} \cdot \mathrm{g}^{-1}, 152 \mathrm{~m}^{2} \mathrm{~g}^{-1}$ and $0.13 \mathrm{~cm}^{3} \cdot \mathrm{g}^{-1}$, respectively (Table 1 ). Figure 4 showed the $\mathrm{N}_{2}$ adsorption isotherms of MSGx and their comparative $\mathrm{Mx}$ samples. MSG0.5 and MSG2.5 had the type IV isotherm and an obvious hysteresis loop at $\mathrm{p} / \mathrm{p}_{0}$ of $0.4 \sim 0.5$, further proving the epitaxially grown shell with mesoporous structure. Unlike MSG0.5 and MSG2.5 exhibited similar isotherms, M0.5 and M2.5 had the diverse isotherms (Fig. 4). Due to the influence of xerogel's hydrophobic surface, MSGx composites had the structure and symmetry different from their corresponding Mx, but similar to the mesoporous silica with the hollow structure formed on oily microemulsion droplets $[27,28]$.

Figure 5 presents the FT-IR spectra of SG, MSGx and Mx samples. C-H stretching vibration and bending bands appeared in the spectrum of SG and MSGx samples at 2977 and $1285 \mathrm{~cm}^{-1}$, respectively, which illustrated the methyl functional groups [29] that lead to the hydrophobicity of silicic xerogel. The band at $800 \mathrm{~cm}^{-1}$ on these samples was ascribed to the bond of Si-C [30]. Si-OH vibration band at $968 \mathrm{~cm}^{-1}$ appeared in $\mathrm{Mx}$ but absent on SG and MSGx samples, since the Si-O- functional groups of MTMS and DMDM precursors had been consumed in the completed polycondensation to prepare 
silicic xerogel, while the $\mathrm{Si}-\mathrm{OH}$ resulting from the shell of mesoporous silica could be neglected to some extent. Likewise, the band at $1070 \mathrm{~cm}^{-1}$ ascribed to Si-O-Si asymmetric stretching bond [29] was reasonably strong on the spectrum of Mx but weak on that of SG sample; for MSGx composite, its band at $1100 \mathrm{~cm}^{-1}$ was slightly stronger than that of SG xerogel because of the mesoporous silica coated on its surface. MSGx composite exhibited the characteristics of both SG and Mx since the shell failed to wrap the core completely so that the SG core was exposed in many cracks (Fig. S8).

As demonstrated in Figure S9, there were two groups of peak on the ${ }^{29}$ Si NMR spectrum of SG sample: the peak at -21.7 ppm plus a shoulder at $-12.4 \mathrm{ppm}$ was ascribed to the silicon connected with two carbon and two oxygen atoms, while the one at $-67.5 \mathrm{ppm}$ plus a shoulder at $-57.7 \mathrm{ppm}$ was assigned to $\mathrm{T} 3[\mathrm{RSi}(\mathrm{OSi}) 3]$ and $\mathrm{T} 2$ [RSi(OSi)2OH] resonances [31], respectively. Of course these peaks were absent on the mesoporous silica sample M0.5 where the strong signal at $-101 \mathrm{ppm}$ with two shoulders at -92 and $-110 \mathrm{ppm}$ respectively was attributed to $\mathrm{Q} n$ [Si(OSi) $n(\mathrm{OH}) 4-n]$ species [31], i.e., isolatedsilanol sites, $(\mathrm{SiO})_{3} * \mathrm{SiOH}\left(\mathrm{Q}^{3},-101 \mathrm{ppm}\right),(\mathrm{SiO})_{4} * \mathrm{Si}\left(\mathrm{Q}^{4},-110 \mathrm{ppm}\right)$ [32]. MSG0.5 and MSG2.5 composites obviously exhibited the characteristic of SG, but the signal of silica was very weak and only some prominences appeared in the range from -85 to -110 ppm (Fig. S9b, S9c).

\subsection{Adsorption of nitrosamines in tobacco extract solution}

SG xerogel was inferior to SBA-15 in the liquid adsorption of TSNA at the low S/L ratio of 0.5 because SG trapped $15 \%$ of TSNA while SBA-15 captured $20.4 \%$. 
However, the advantage of super hydrophobic xerogel emerged once the S/L ratio achieved 1.0 on which SG adsorbed $41 \%$ while SBA-15 trapped $29.5 \%$. Further increase of S/L enabled SG to exhibit a capacity 50\% higher than that of SBA-15 (Fig. 1b). If the adsorption capability of sorbent was calculated by its surface area, the value of SG would be 160 times higher than that SBA-15 (Table 2), since the surface area of SG $\left(4 \mathrm{~m}^{2} \mathrm{~g}^{-1}\right)$ is about $0.4 \%$ of that of SBA-15 $\left(918 \mathrm{~m}^{2} \mathrm{~g}^{-1}\right.$, Table 1$)$.

Table 1 lists the adsorption of nitrosamines in tobacco exact solution by porous sorbents. Microporous zeolite ZSM-5 and Y captured 25 31\% of nitrosamines in the solution at the S/L of 1 owing to the complex composition of tobacco solution $[1,2]$. The large pore size of zeolite $\mathrm{Y}$ displayed a minor advantage, but acidic zeolite HZSM-5 was slightly superior to its basic analogue NaZSM-5 due to the attraction of proton towards the N-NO group of nitrosamines [20]. Under the same conditions, mesoporous silica MCM-41, SBA-15 and MCM-48 trapped 29 41\% of nitrosamines in solution. The narrow channels of MCM-48 plus the special double helix structure was beneficial for the adsorption, because the cross section of nitosamines usually smaller than $1 \mathrm{~nm}$ [1]. Another two mesoporous silica sorbents with relatively narrow channels, M0.5 and M2.5, could adsorb 36 43\% of nitrosamines in solution. Amorphous activated carbon (AC) captured $34 \%$ of nitrosamines, which was slightly less than that by SG though AC has a large surface area (Table 1). Coating mesoporous silica on SG enables the sorbent to exert a high efficiency; MSG0.5 adsorbed 59\% of nitrosamines while MSG2.5 trapped 54\%, exceeding all other porous samples. Besides, they also 
exhibited the high activity in adsorption of TSNA in tobacco solution, and about $30 \%$ of the carcinogens were removed. Capture of TSNA in tobacco extract solution is very hard because the bulky TSNA molecule is difficult to be adsorbed but easy to desorb [2]. SBA-15, MCM-41 and MCM-48 showed a similar capability (about 20\%, Table 1), almost same as that of zeolite $\mathrm{NaY}$, while the activity of AC was same as that of M0.5. Super hydrophobic xerogel SG trapped the least of TSNA due to its very small surface area, while zeolite ZSM-5 exhibited a surprising high capability of capturing TSNA in tobacco extract solution owing to its special pore size $(0.54-0.56 \mathrm{~nm})$ and hydrophobility that originated from its high $\mathrm{Si} / \mathrm{Al}$ ratio [2]. Most sorbents trapped less TSNA than nitrosamines in tobacco extract solution, since TSNA is part of the nitrosamines detected by spectrophotometric method $[2,20]$, but NaZSM-5 was the only exception on which two values were similar.

Some strange phenomena emerged in the removal of TSNA (Table 2). Firstly, SG and its derivates MSGx possessed the highest value of TSNA removal calculated either by weight (value B) or by surface area (value C), and SG had the maximum of $8.2 \mathrm{nmol}$ $\mathrm{m}^{-2}$, which tens time exceeded that of other samples (Table 2), while MSGx showed the data $6 \sim 10$ times higher than that of mesoporous silica. Secondly, the efficiency of microporous zeolite, calculated by surface area, was double higher than that of common mesoporous silica samples and AC. Thirdly, ZSM-5 zeolites exhibited a kind of selectivity towards 4-( $\mathrm{N}$-nitrosomethylamino)-1-(3-pyridyl)-1- butanone (NNK) among three components of TSNA: the original molar ratio of $N^{\prime}$-nitrosonornicotine (NNN): 
$N^{\prime}$-nitrosoanatabine (NAT):NNK in the solution was $1.5: 2.3: 1$, yet the trapped TSNA by NaZSM-5 was 0.9:1.5:1, and that by HZSM-5 was 0.6:1.2:1 (calculated from the data in Table 2). One reason for the tendentious adsorption of NNK is the good match between the narrow zig-zag channels of ZSM-5 zeolite and the spindly shape of NNK molecule, but further investigation is required. SG sample equably trapped three components of TSNA and the removal of NNN: NAT: NNK was same (13\%). Coating mesoporous silica on SG enabled the sorbent to capture more NAT, say, 3\% more on MSG0.5 composite and 6\% more on MSG2.5 (Table 2), similar to that on MCM-41, SBA-15 and MCM-48 on which about $5 \%$ more of NAT to be trapped than NNN or NNK. .

\section{Discussion}

Two factors are necessary for liquid adsorption of TSNA. One is the attraction towards the target, and another is the optimal micro-environment suitable for adsorption. For the former the electrostatic interaction between the cations of zeolite and the negative charged N-NO group of nitrosamines $[2,20]$ are beneficial, while for the latter the channels or long thin pores in sorbent are important because they can direct adsorbate to adsorptive site, enhancing the sorption process [33]. Super hydrophobocity endows SG sample the high efficiency of capturing TSNA, and it trapped the TSNA of $32.8 \mathrm{nmol}$ $\mathrm{g}^{-1}$ though its BET specific surface area is only $4 \mathrm{~m}^{2} \mathrm{~g}^{-1}$. That means, the surface of SG sample is very active to capture TSNA in tobacco solution $\left(8.2 \mathrm{nmol} \mathrm{m}^{-2}\right)$. However, SG xerogel floated on water, which limited the contact of sorbent with solution hence modification of SG is necessary. Three advantages were obtained after the silicic 
xerogel was coated with mesoporous silica through epitaxial growth process. Firstly, the surface area of sorbent is enlarged tens time (from 4 to $150 \sim 200 \mathrm{~m}^{2} \mathrm{~g}^{-1}$ ). Secondly, part surface became less hydrophobic but somewhat hydrophilic so that the sorbent could immerse tobacco solution to contact with fluid. Thirdly, porous surface was built on the sorbent. SG itself is imporous but MSG0.5 composite had the most probable pore size of $3.8 \mathrm{~nm}$ and MSG2.5 had the one of $3.5 \mathrm{~nm}$ (Fig. 4A).

SG sample and its derive MSGx had a very high value $\mathrm{C}$ in liquid adsorption of TSNA (Table 2). MSGx composites kept the hydrophobic core but combined the mesoporous shell hence they had a larger adsorption capacity than silicic xerogel and mesoporous silica did. The exposed silicic xerogel in the composite exhibited the super-hydrophobicity to attract oily molecules TSNA, providing the driving force to pull the target carcinogen; while the mesoporous shell enables the sorbent to contact with more TSNAs and to limit tardily the thermal motion of adsorbates [33], forming a synergy to promote the liquid adsorption of TSNA. Besides, fabrication of core-shell structure through epitaxial growth makes mesoporous silica tightly combined with inner silicic xerogel, forming the convenient monolith sorbent and offering a clue for design new functional materials.

\section{Conclusion}

The super-hydrophobic silicic xerogel (SG) synthesized by MTMS and DMDMS offers a powerful sorbent to remove the oily carcinogen nitrosamines in tobacco extract solution. 
Mesoporous silica is successfully introduced to the super-hydrophobic silicic xerogel under weak acid system, forming mesoporous shell with a thickness of 100 300 nm. The composite MSGx has hydrophobic core of silicic xerogel and the somewhat hydrophilic shell of mesoporous silica, exhibiting an enhanced activity in capture of nitrosamines and TSNA in tobacco extract solution. Epitaxial growth strategy is practical for fabrication of new functional materials.

\section{Acknowledgment}

NSF of China (21173117 and 21273106), Analysis Center of Nanjing University financially supported this research.

\section{References}

[1] F. Wei, J.Y. Yang, L. Gao, F.N. Gu, J.H. Zhu, J. Hazard. Mater.172 (2009), $1482-1490$.

[2] W.G. Lin, B.C. Huang, B. Zhou, X.L. Lv, L.J. Wang, S.J. Zheng, W.M. Wang, Y.G. Tong, Y. Wang, J.H. Zhu, J. Porous Mater. 21 (2014), 311-320.

[3] X.D. Sun, W.G. Lin, L.J. Wang, B. Zhou, X.L. Lv, Y.Wang, S.J. Zheng, W.M. Wang, Y.G. Tong, J.H. Zhu, Microporous Mesoporous Mater.200 (2014), 260-268.

[4] S. Tatsuoka, Patent No. EP1782702 A1 (2005).

[5] Z.X. Xue, S.T. Wang, L. Lin, L. Chen, M.J. Liu, L. Feng, L. Jiang, Adv. Mater. 23 (2011), 4270-4273.

[6] H.W. Liang, Q.F. Guan, L.F. Chen, Z. Zhu, W.J. Zhang, S.H. Yu, Angew. Chem. 124 (2012), 5191-5195. 
[7] J.K. Yuan, X.G. Liu, O. Akbulut, J.Q. Hu, S.L. Suib, J. Kong, F. Stellacci, Nat. Nanotechnol. 3 (2008), 332-336.

[8] J. Lahann, Nat. Nanotechnol.3 (2008), 320-321.

[9] J.T. Korhonen, M.Kettunen, R.H.A. Ras, O. Ikkala, ACS Appl. Mater. Interfaces. 3 (2011), 1813-1816.

[10] A. Tuteja, W. Choi, M. Ma, J.M. Mabry, S.A. Mazzella, G.C. Rutledge, G.H. McKinley, R.E. Cohen, Science 318 (2007), 1618-1622.

[11] J.P. Zhang, S. Seeger, Adv. Funct. Mater. 21 (2011), 4699-4704.

[12] M. Nicolas, F. Guittard, S. Geribaldi, Angew. Chem.118 (2006), 2309-2312.

[13] A.V. Rao, N.D. Hegde, H. Hirashima, J. Colloid Interface Sci.305 (2007), 124-132.

[14] J. G. Reynolds, P.R. Coronado, L.W. Hrubesh, Energy Sources. 23 (2001), 831-843.

[15] M. Ma, R.M. Hill, Curr. Opin. Colloid Interface Sci. 11 (2006), 193-202.

[16] K. Kanamori, Y. Kodera, G. Hayase, K. Nakanishi, T. Hanada, J. Colloid Interface Sci. 357 (2011), 336-344.

[17] K. Kanamori, M. Aizawa, K. Nakanishi, T. Hanada, Adv. Mater. 19 (2007), $1589-1593$.

[18] G. Hayase, K. Kanamori, K. Nakanishi,J. Mater. Chem. 21 (2011),17077-17079.

[19] R.R. Baker, Smoke chemistry. In: Layten D, Nielsen MT (ed). Tobacco: production, chemistry and technology. Blackwell Science, Oxford, 1999, pp398-439. [20] Y. Xu, J.H. Zhu, L.L. Ma, A. Ji, Y.L. Wei, X.Y. Shang, Microporous Mesoporous 
Mater. 60 (2003), 125-138.

[21] D.Y. Zhao, J.L. Feng, Q.S. Huo, N. Melosh, G.H. Fredrickson, B.F. Chmelka, G.D. Stucky, Science. 279(1998), 548-552.

[22] S.C. Shen, X.Y. Chen, S. Kawi, Langmuir 20 (2004), 9130-9137.

[23] K. Schumacher, P.I. Ravikovitch, A.D. Chesne, A.V. Neimark, K.K. Unger, Langmuir 16 (2000), 4648-4654.

[24] K.A. Wagner, N.H. Finkel, J.E. Fossett, I.G. Gillman, Anal. Chem. 77 (2005), 1001-1006.

[25] K. Nakanishi, J. Porous Mater. 4 (1997), 67-112.

[26] Y. Zhou, K. Li, J.Y. Yang, C.X. Guan, Y. Wang, C.J. Liu, J.H. Zhu, Small 8 (2012), 1373-1383.

[27] A.F. Zhang, Y.C. Zhang, N. Xing, K.K. Hou, X. W. Guo, Chem. Mater. 21 (2009), $4122-4126$

[28] X. Yang, S.J. Liao, J.H. Zeng, Z.X. Liang, Appl. Sur. Sci. 257 (2011), 4472-4477.

[29] A.V.Rao, S.S. Latthe, D.Y. Nadargi, H. Hirashima, V. Ganesan, J. Colloid. Interface Sci. 332 (2009), 484-490.

[30] N. Hering, K. Schriber, R. Riedel, O. Lichtenberger, J. Woltersodorf, Appl. Organomet. Chem. 15 (2001), 879-886.

[31] W. Guo, J.Y. Park, M.O. Oh,H.W. Jeong, W.J. Cho, I. Kim, C.S. Ha, Chem. Mater. 15 (2003), 2295-2298.

[32] X.S. Zhao, G.Q. Lu,A.K. Whittaker, G.J. Millar, H.Y. Zhu, J. Phys. Chem. B. 101 
(1997), 6525-6531.

[33] S.J. Reitmeier, O.C. Gobin,A. Jentys, J.A. Lercher, Angew. Chem. Int. Ed. 48 (2009), 533-538. 
Table 1. The texture properties and nitrosamines removal of SG and porous sorbents.

\begin{tabular}{|c|c|c|c|c|c|c|}
\hline Samples & $\begin{array}{c}\mathrm{S}_{\mathrm{BET}} \\
\left(\mathrm{m}^{2} \cdot \mathrm{g}^{-1}\right)\end{array}$ & $\begin{array}{c}\mathrm{V}_{\mathrm{p}} \\
\left(\mathrm{cm}^{3} \cdot \mathrm{g}^{-1}\right)\end{array}$ & $\begin{array}{c}\mathrm{D}_{\mathrm{p}} \\
(\mathrm{nm})\end{array}$ & $\mathrm{Si} / \mathrm{Al}$ & $\begin{array}{c}\text { Removal of } \\
\text { nitrosamines (\%) }\end{array}$ & $\begin{array}{l}\text { Removal of } \\
\text { TSNA (\%) }\end{array}$ \\
\hline $\mathrm{SG}^{\mathrm{a}}$ & 4 & 0.003 & - & - & 37.7 & 13.1 \\
\hline $\operatorname{MSG} 0.5^{\mathrm{b}}$ & 208 & 0.16 & 3.8 & - & 59.1 & 30.9 \\
\hline MSG2.5 & 152 & 0.13 & 3.5 & - & 54.5 & 28.9 \\
\hline $\mathrm{M} 0.5^{\mathrm{c}}$ & 1078 & 0.77 & 2.1 & - & 43.2 & 15.7 \\
\hline M2.5 & 576 & 0.56 & 3.4 & - & 36.6 & - \\
\hline NaZSM-5 & 354 & 0.11 & 0.5 & 12.5 & 25.0 & 28.8 \\
\hline HZSM-5 & 346 & 0.10 & 0.5 & 12.5 & 30.8 & 24.2 \\
\hline $\mathrm{NaY}$ & 766 & 0.31 & 0.7 & 2.3 & 31.8 & 18.2 \\
\hline MCM-41 & 1342 & 1.01 & 2.8 & - & 41.8 & 19.1 \\
\hline SBA-15 & 918 & 1.18 & 8.5 & - & 29.5 & 20.3 \\
\hline MCM-48 & 1124 & 0.75 & 2.3 & - & 40.6 & 19.5 \\
\hline $\mathrm{AC}$ & 1128 & 0.54 & $\sim 1.0^{\mathrm{d}}$ & - & 34.3 & 15.7 \\
\hline
\end{tabular}

${ }^{\text {a }} \mathrm{SG}$ is silicic xerogel.

${ }^{\mathrm{b}}$ MSG is silicic xerogel covered with mesoporous silica.

${ }^{\mathrm{c}} \mathrm{M} 0.5$ is the mesoporous silica synthesized without adding SG. ${ }^{\mathrm{d}}$ The primary pore size of AC. 
Table 2. Residual TSNA in the tobacco extract solution adsorbed by porous sorbents

\begin{tabular}{|c|c|c|c|c|c|c|c|c|c|c|}
\hline Sample & $\begin{array}{l}\text { NNN } \\
(\text { nmol }\end{array}$ & $\begin{array}{l}\text { Cut } \\
(\%)\end{array}$ & $\begin{array}{l}\text { NAT } \\
\left(\mathrm{nmolL}^{-1}\right)\end{array}$ & $\begin{array}{l}\text { Cut } \\
(\%)\end{array}$ & $\begin{array}{l}\text { NNK } \\
\text { ( nmol }\end{array}$ & $\begin{array}{l}\text { Cut } \\
(\%)\end{array}$ & $\begin{array}{l}\text { TSNA } \\
\text { ( nmol }\end{array}$ & ( nmol & $\begin{array}{l}\text { Reduced } \\
\text { (nmol } \\
\left.\mathrm{g}^{-1}, \mathrm{~B}\right)\end{array}$ & $\begin{array}{l}(\mathrm{nmol} \\
\left.\mathrm{m}^{-2}, \mathrm{C}\right)\end{array}$ \\
\hline & $\left.\mathrm{L}^{-1}\right)$ & & & & $\left.\mathrm{L}^{-1}\right)$ & & $\left.\mathrm{L}^{-1}\right)$ & $\left.\mathrm{L}^{-1}, \mathrm{~A}\right)$ & & \\
\hline Control & 39.0 & - & 59.8 & - & 26.1 & - & 124.9 & - & - & - \\
\hline NaZSM-5 & 29.4 & 24.6 & 43.9 & 26.5 & 15.9 & 38.8 & 89.2 & 35.7 & 71.4 & 0.20 \\
\hline $\mathrm{NaY}$ & 31.1 & 20.3 & 48.7 & 18.6 & 22.2 & 14.8 & 102.0 & 22.9 & 45.8 & 0.06 \\
\hline HZSM-5 & 32.8 & 15.9 & 47.6 & 20.4 & 15.0 & 42.6 & 95.4 & 29.5 & 59.0 & 0.17 \\
\hline MCM-41 & 31.6 & 18.8 & 46.0 & 23.0 & 23.2 & 11.1 & 100.8 & 24.1 & 48.2 & 0.04 \\
\hline SBA-15 & 31.6 & 18.8 & 46.0 & 23.0 & 21.7 & 16.7 & 99.3 & 25.6 & 51.2 & 0.06 \\
\hline MCM-48 & 31.6 & 18.8 & 46.6 & 22.1 & 22.2 & 14.8 & 100.4 & 24.5 & 49.0 & 0.04 \\
\hline $\mathrm{AC}$ & 33.9 & 13.0 & 47.1 & 21.2 & 21.7 & 16.7 & 102.7 & 22.2 & 44.4 & 0.04 \\
\hline SG & 33.9 & 13.0 & 51.9 & 13.3 & 22.7 & 13.0 & 108.5 & 16.4 & 32.8 & 8.2 \\
\hline MSG0.5 & 27.7 & 29.0 & 40.2 & 32.7 & 18.8 & 29.6 & 86.3 & 38.6 & 77.2 & 0.37 \\
\hline MSG2.5 & 28.8 & 26.1 & 40.2 & 32.7 & 19.3 & 26.0 & 88.3 & 36.6 & 73.2 & 0.48 \\
\hline M0.5 & 32.2 & 17.4 & 49.7 & 16.8 & 23.2 & 11.1 & 105.1 & 19.8 & 39.6 & 0.04 \\
\hline
\end{tabular}

${ }^{a}$ NNN is N'-Nitrosonornicotine.

${ }^{\mathrm{b}} \mathrm{NAT}$ is $\mathrm{N}^{\prime}$-Nitrosoanatabine.

${ }^{\mathrm{c}}$ NNK is 4-(N- Nitrosomethylamino)-1-(3- Pyridyl)-1- Butanone.

${ }^{\mathrm{d}}$ TSNA is tobacco specific nitrosamines. 


\section{Caption of Figures}

Scheme 1. Schematic illustration of epitaxial growth of mesoporous silica on the surface of silicic xerogel along with the adsorption of TSNA.

Figure 1. Adsorption of (a) water and (b) TSNA by SG and SBA-15 samples .

Figure 2. The SEM images of the xerogel matrix SG and its epitaxially grown sample MSGx.

Figure 3. The TEM images of the xerogel matrix (a, b) SG and its epitaxially grown samples (c, d) MSG0.5 and (e, f) MSG2.5.

Figure 4. $\mathrm{N}_{2}$ adsorption-desorption isotherm of (A) MSGx and (B) Mx samples

Figure 5. FT-IR spectra of SG, MSGx and Mx samples. 


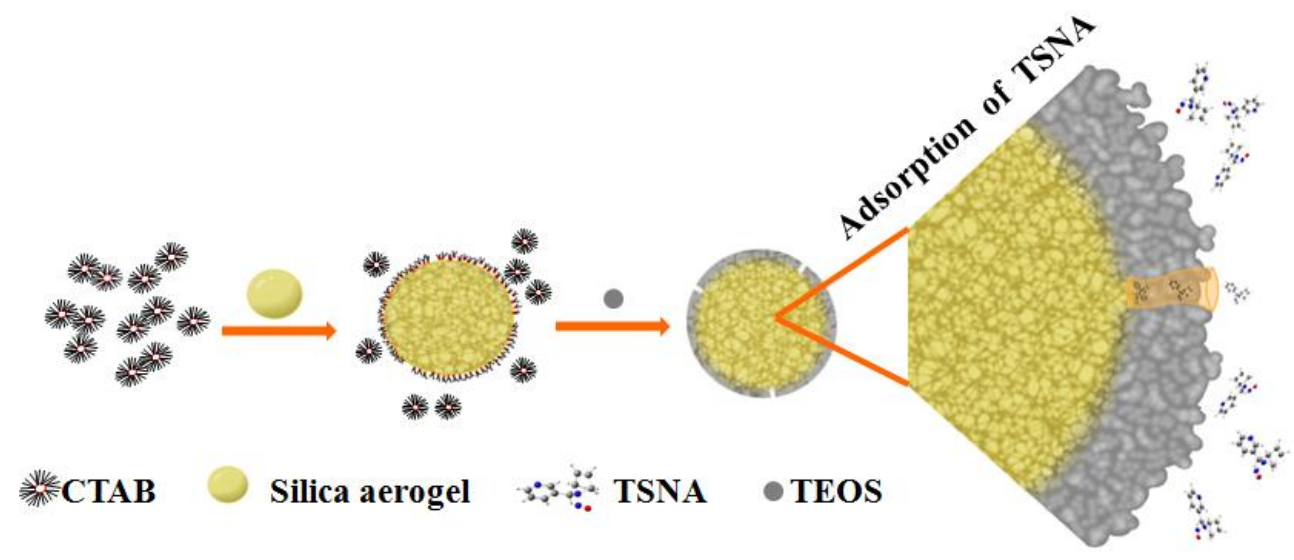

Scheme 1.Schematic illustration of epitaxial growth of mesoporous silica on the surface of silicic xerogel along with the adsorption of TSNA.
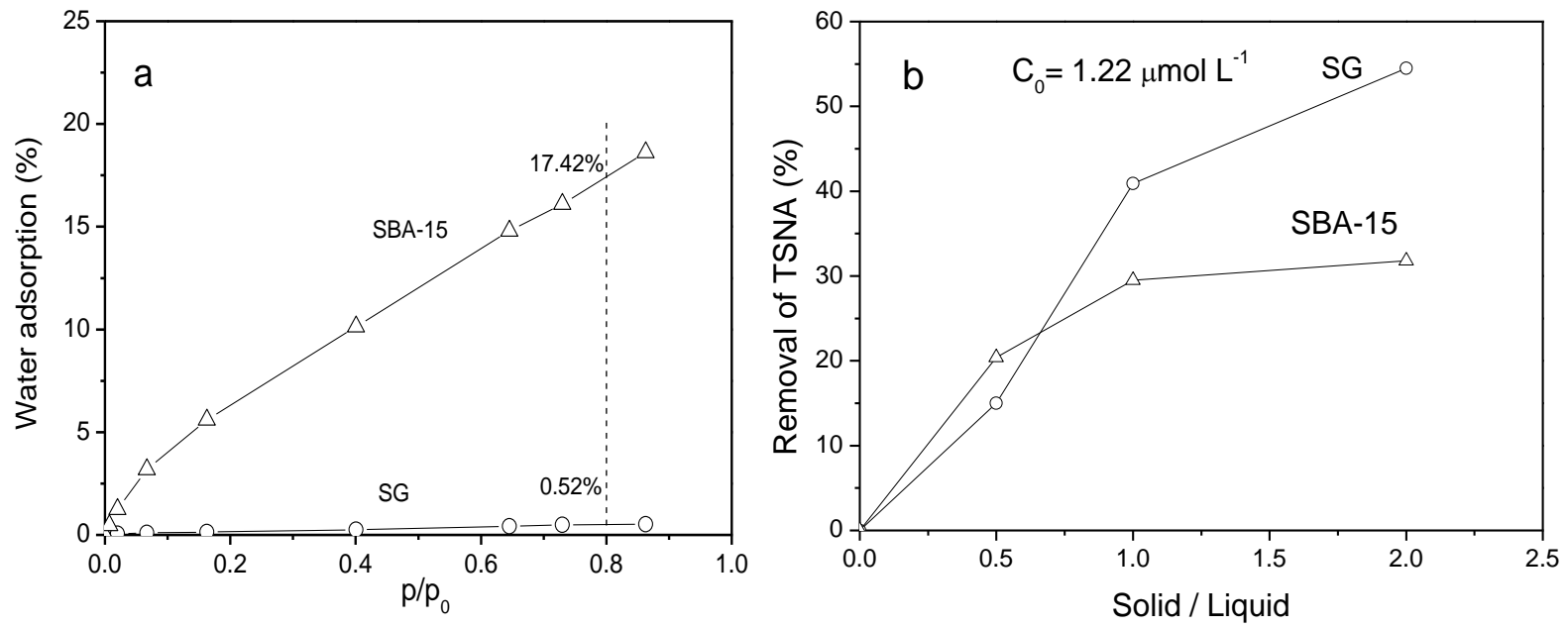

Figure 1. Adsorption of (a) water and (b) TSNA by SG and SBA-15 samples . 

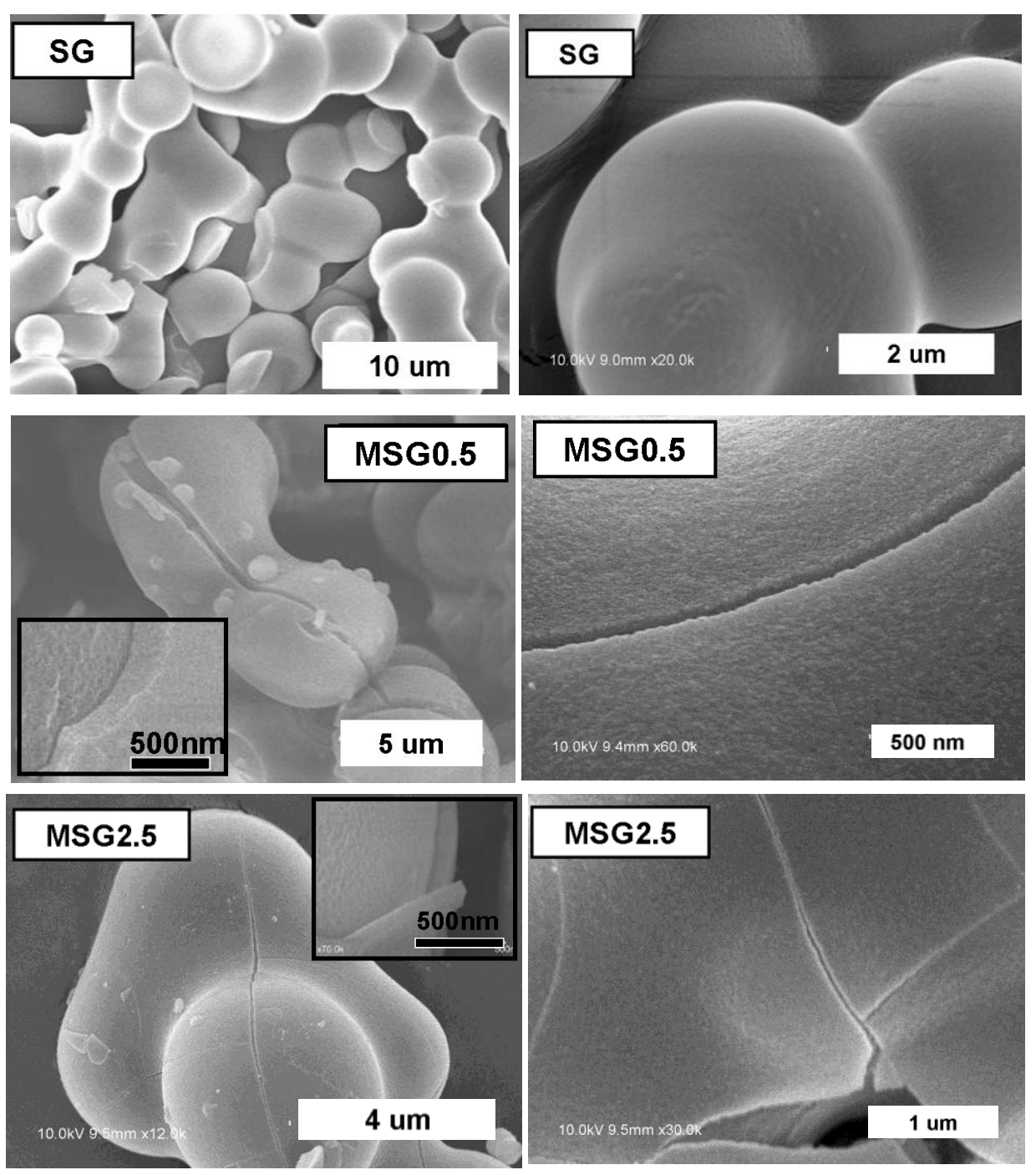

Figure 2.The SEM images of the xerogel matrix SG and its epitaxially grown sample MSGx. 

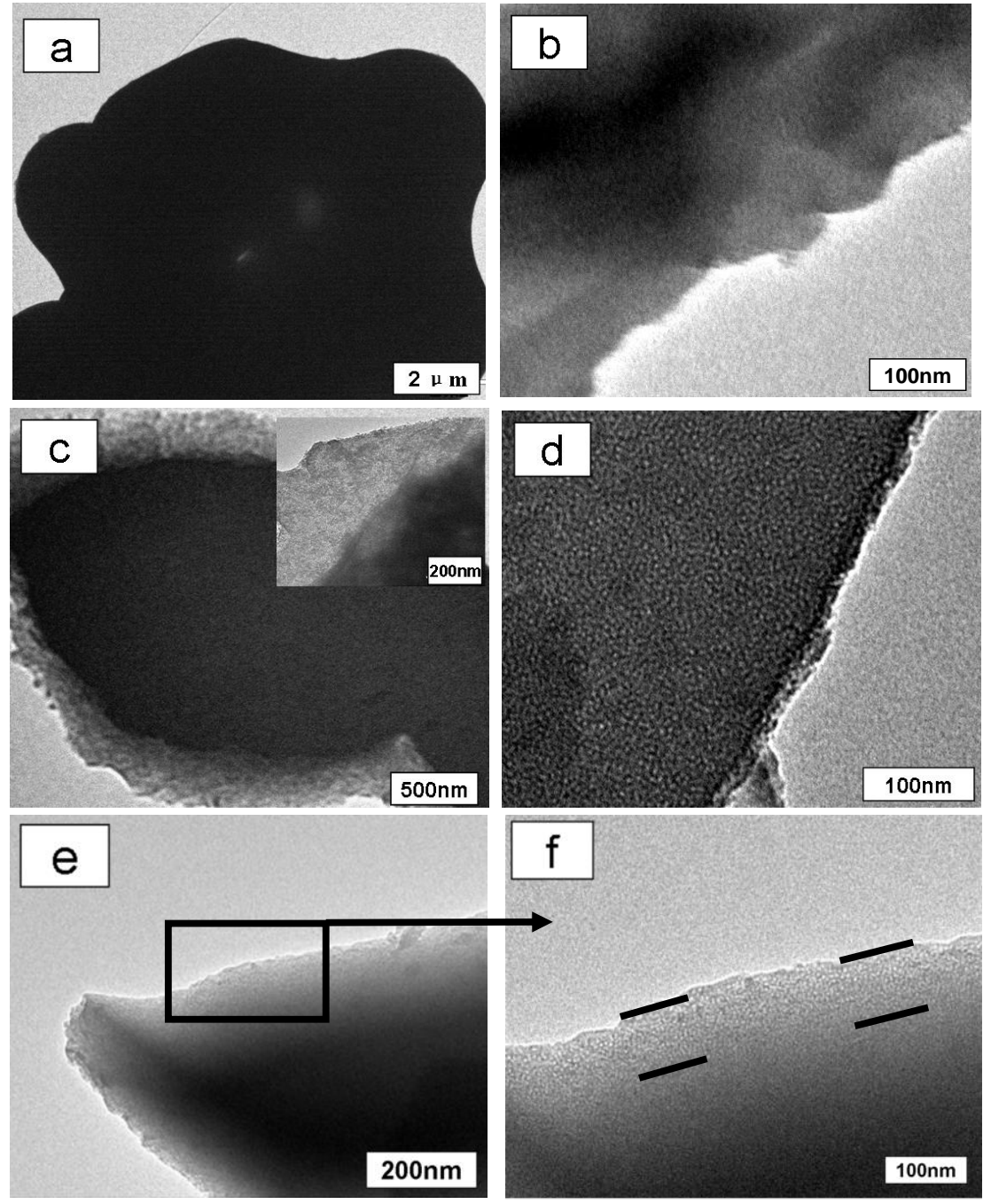

Figure 3.The TEM images of the xerogel matrix (a, b) SG and its epitaxially grown samples (c, d) MSG0.5 and (e, f) MSG2.5. 

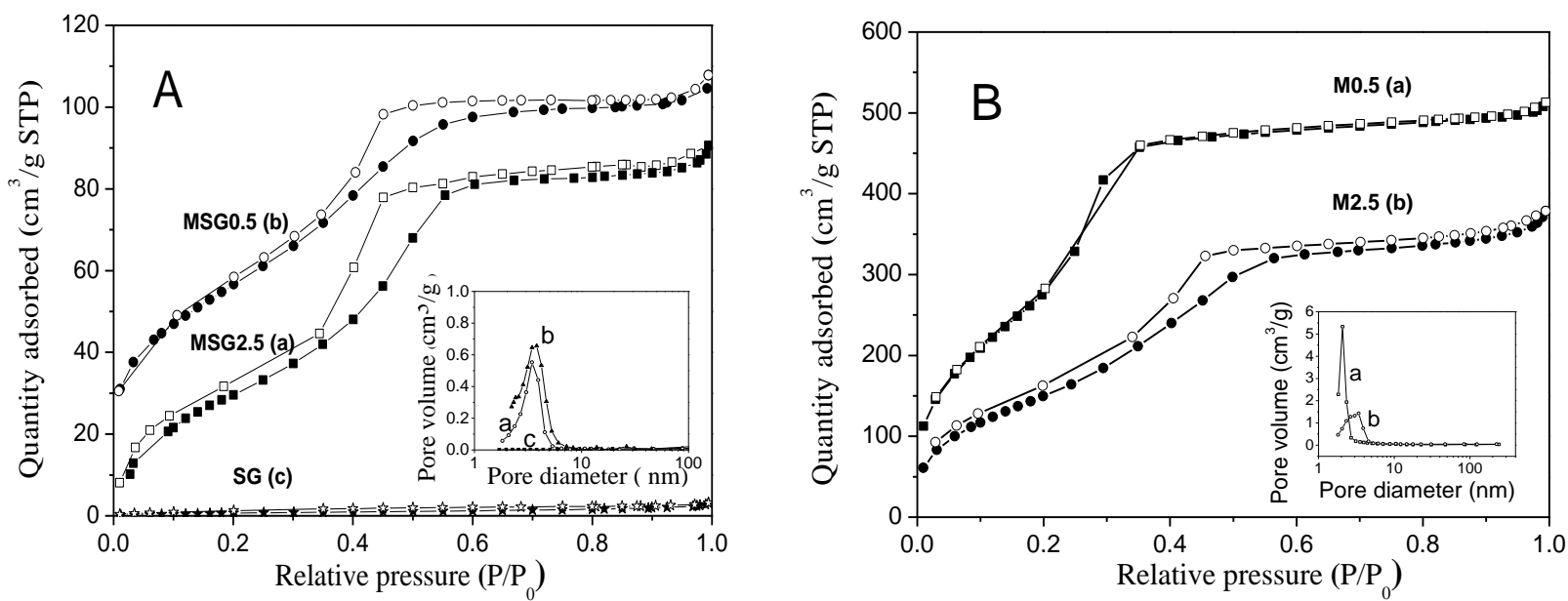

Figure 4. $\mathrm{N}_{2}$ adsorption-desorption isotherm of (A)MSGx and (B)Mx samples 


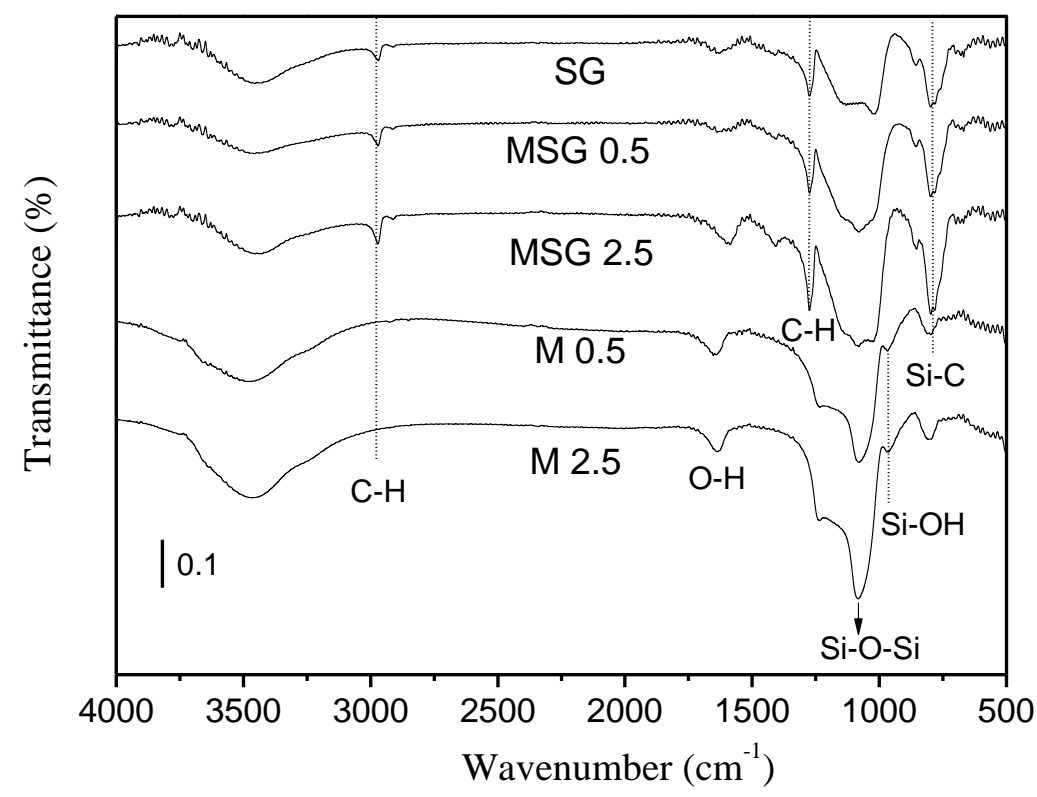

Figure 5.FT-IR spectra of SG, MSGx and Mx samples. 
Graphical Abstract

\section{Capturing nitrosamines in aqueous solution by composited super-hydrophobic silicic xerogel}

Shuo Hao Li, Wei Gang Lin, Bi Cheng Huang, Lei-jun Wang, Wen-bo Gu, Wei-miao Wang, Zheng Yu Yang, Ying Wang, Jian Hua Zhu

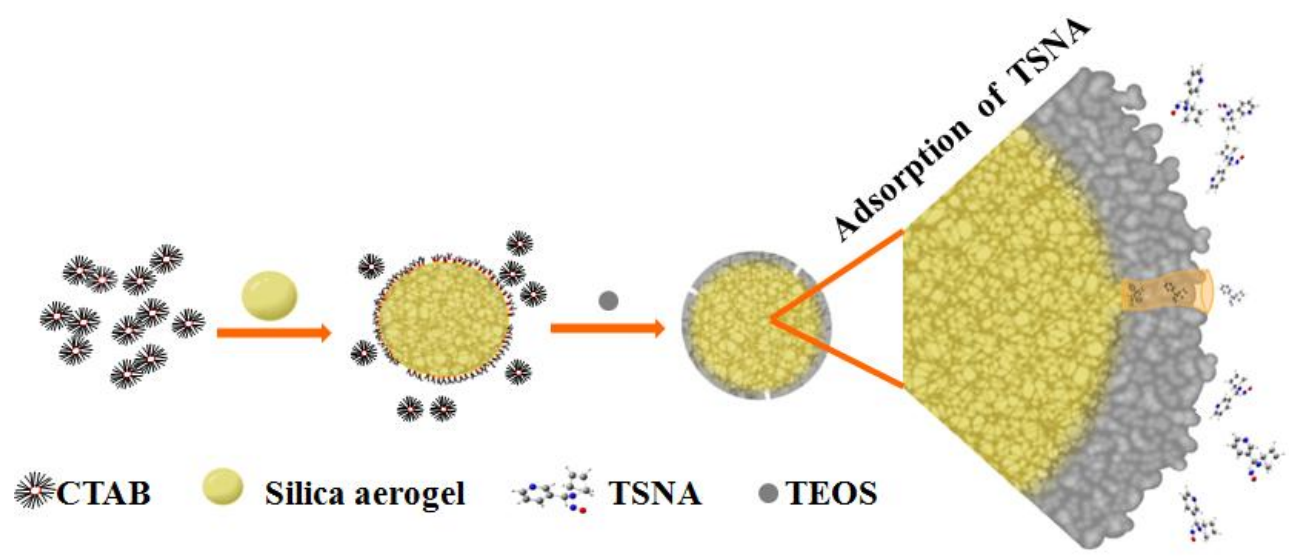

Scheme 1. Schematic illustration of epitaxial growth of mesoporous silica on the surface of silicic xerogel along with the adsorption of TSNA. 